\title{
THE FOCUS-CENTER-LIMIT CYCLE BIFURCATION IN SYMMETRIC 3D PIECEWISE LINEAR SYSTEMS*
}

\author{
EMILIO FREIRE ${ }^{\dagger}$, ENRIQUE PONCE ${ }^{\dagger}$, AND JAVIER ROS ${ }^{\dagger}$
}

\begin{abstract}
The birth of limit cycles in 3D (three-dimensional) piecewise linear systems for the relevant case of symmetrical oscillators is considered. A technique already used by the authors in planar systems is extended to cope with 3D systems, where a greater complexity is involved.

Under some given nondegeneracy conditions, the corresponding theorem characterizing the bifurcation is stated. In terms of the deviation from the critical value of the bifurcation parameter, expressions in the form of power series for the period, amplitude, and the characteristic multipliers of the bifurcating limit cycle are also obtained.

The results are applied to accurately predict the birth of symmetrical periodic oscillations in a 3D electronic circuit genealogically related to the classical Van der Pol oscillator.
\end{abstract}

Key words. piecewise linear systems, bifurcation theory, limit cycles

AMS subject classifications. $37 \mathrm{G} 15,34 \mathrm{C} 15$

DOI. $10.1137 / 040606107$

1. Introduction and main results. Piecewise linear modeling of nonlinear dynamical systems is especially successful in some engineering problems, such as the analysis and design of electronic oscillators or control systems (see, e.g., [CFPT02]). However, in the framework of piecewise linear systems, there are no general bifurcation results explaining the appearance or disappearance of self-sustained oscillations, as is the case for the Hopf bifurcation theorem in the context of differentiable systems. Thus, the authors gave in [FPR99] a complete characterization of the focus-centerlimit cycle bifurcation for symmetric planar piecewise linear systems. Now we show how the corresponding result can be extended to the 3D case.

We consider a common situation in applications, namely, dynamical systems defined by piecewise continuous vector fields with three linear zones and two parallel frontiers. Furthermore, it is assumed that such systems show symmetry with respect to the origin; that is, if we put them in the form $d \mathbf{x} / d \tau=f(\mathbf{x})$ with $\mathbf{x} \in \mathbb{R}^{3}$, they satisfy $f(-\mathbf{x})=-f(\mathbf{x})$. In particular, $f(0)=0$, and so the origin is an equilibrium point for all values of the parameters. By means of a linear change of variables, it is always possible to suppose that the frontiers are the planes $\Sigma_{1}=\left\{\mathbf{x} \in \mathbb{R}^{3}: x_{1}=1\right\}$ and $\Sigma_{-1}=\left\{\mathbf{x} \in \mathbb{R}^{3}: x_{1}=-1\right\}$. We denote by $L$ (left), $C$ (central), and $R$ (right) the regions of $\mathbb{R}^{3}$ at which $x_{1}<-1,\left|x_{1}\right| \leq 1$, and $x_{1}>1$, respectively, hold.

To be more precise, we consider systems expressed as follows:

$$
\dot{\mathbf{x}}= \begin{cases}A_{L} \mathbf{x}-\mathbf{b} & \text { if } x_{1}<-1, \\ A_{C} \mathbf{x} & \text { if }\left|x_{1}\right| \leq 1, \\ A_{L} \mathbf{x}+\mathbf{b} & \text { if } x_{1}>1\end{cases}
$$

\footnotetext{
*Received by the editors April 1, 2004; accepted for publication (in revised form) February 2, 2005; published electronically August 3, 2005. This research was partially supported by grants DPI2000-1218-C04-04, BFM2001-2668, and BFM2003-00336 of the Spanish Ministry of Science and Technology. The authors were also supported by Junta de Andalucía, as members of the research group TIC-130, in the budget corresponding to 2003.

http://www.siam.org/journals/siap/65-6/60610.html

${ }^{\dagger}$ Departamento Matemática Aplicada II, Universidad de Sevilla, Escuela Superior de Ingenierosm, Camino de los Descubrimientos s/n, 41092, SEVILLA, Spain (emilio@ma2.us.es, enrique@ma2.us.es, jros@platero.eup.us.es).
} 

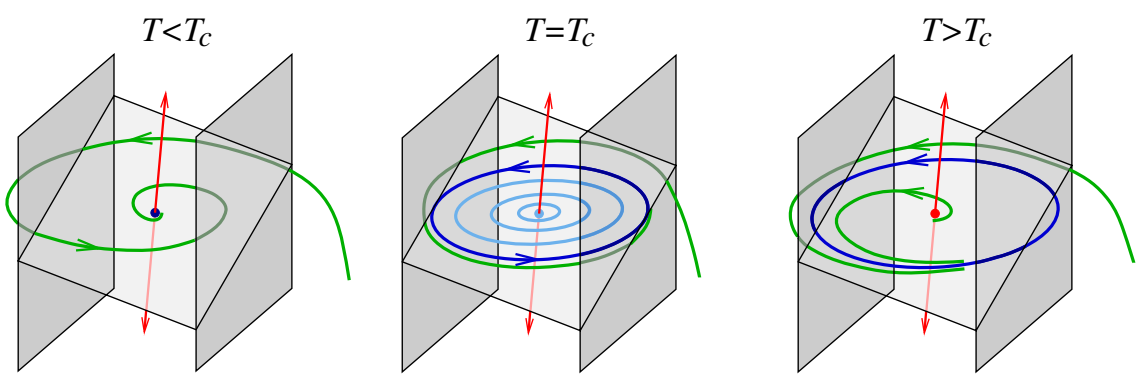

FIG. 1. The focus-center-limit cycle bifurcation in the case $D>0, \gamma>0$. The focal plane and the complementary one-dimensional invariant manifold at the origin are shown, along with the two parallel planes which separate the three linear regions. In the situation sketched, as deduced from Theorem 1.1, the bifurcating limit cycle is of saddle type.

where we have taken advantage of the continuity and symmetry of the vector field involved; in particular, the matrices $A_{L}$ and $A_{C}$ differ only in their first columns.

From Proposition 16 of [CFPT02], under the generic condition of observability, every system (1.1) can be written in the generalized Liénard form

$$
\frac{d}{d \tau}\left[\begin{array}{l}
x_{1} \\
x_{2} \\
x_{3}
\end{array}\right]=\left[\begin{array}{rrr}
t & -1 & 0 \\
m & 0 & -1 \\
d & 0 & 0
\end{array}\right]\left[\begin{array}{l}
x_{1} \\
x_{2} \\
x_{3}
\end{array}\right]+\left[\begin{array}{c}
T-t \\
M-m \\
D-d
\end{array}\right] \operatorname{sat}\left(x_{1}\right),
$$

where $\operatorname{sat}\left(x_{1}\right)$ is the normalized saturation

$$
\operatorname{sat}\left(x_{1}\right)=\left\{\begin{array}{cc}
-1, & x_{1} \leq-1 \\
x_{1}, & \left|x_{1}\right|<1 \\
1, & x_{1} \geq 1
\end{array}\right.
$$

so that, regarding system (1.1), we have

$$
A_{L}=\left[\begin{array}{rrr}
t & -1 & 0 \\
m & 0 & -1 \\
d & 0 & 0
\end{array}\right], \quad A_{C}=\left[\begin{array}{rrr}
T & -1 & 0 \\
M & 0 & -1 \\
D & 0 & 0
\end{array}\right], \quad \mathbf{b}=\left[\begin{array}{c}
T-t \\
M-m \\
D-d
\end{array}\right]
$$

Note that system (1.2) is a particular instance of the more general Lur'e form

$$
\frac{d \mathbf{x}}{d \tau}=A \mathbf{x}+\mathbf{b} \operatorname{sat}\left(\mathbf{c}^{T} \mathbf{x}\right)
$$

for the case $A=A_{L}$ and $\mathbf{c}=\mathbf{e}_{1}$, where $\mathbf{e}_{1}$ stands for the first vector of the canonical basis.

Clearly, the parameters $t, m, d$ and $T, M, D$ stand for the trace, the sum of principal minors of order two, and the determinant of each matrix, and they completely determine the dynamics of the system.

Choosing $T$ as the bifurcation parameter, for the critical value $T_{c}=D / M$ with $M>0$, system (1.2) has a linear center in the zone $C$ (see Figure 1); that is, the matrix $A_{C}$ has a pair of pure imaginary eigenvalues. We want to analyze whether a limit cycle bifurcates from this configuration as the bifurcation parameter $T$ varies. Note the similarities with the classical Hopf bifurcation scenario. 
It will be useful, in order to know the stability of such a limit cycle, to estimate the characteristic multipliers of the limit cycle, that is, the eigenvalues of the derivative of a Poincaré return map defined in an adequate section of the phase space. We will denote the logarithms of these characteristic multipliers by $\mu_{r}$ and $\mu_{a}$, from radial and axial, respectively. Our main result is the following.

THEOREM 1.1. Let us consider system (1.2) with $M>0, T_{c}=D / M$, and $\gamma=D M-D m+d M-t M^{2} \neq 0$. For $T=T_{c}$ the system undergoes a focus-centerlimit cycle bifurcation; that is, from the lineal center configuration in the central zone, which exists for $T=T_{c}$, one limit cycle appears for $\gamma\left(T-T_{c}\right)>0$ and $T-T_{c}$ sufficiently small.

The amplitude " $a$ " (measured as the maximum of $\left|x_{1}\right|$ ), the period $P_{e r}$, and the logarithms of characteristic multipliers $\mu_{r}$ and $\mu_{a}$ of the periodic orbit are analytic functions at 0 , in the variable $\left(T-T_{c}\right)^{1 / 3}$; namely,

$$
\begin{aligned}
a & =1+\frac{(6 \pi)^{2 / 3} M^{4 / 3}}{8 \gamma^{2 / 3}}\left(T-T_{c}\right)^{2 / 3}+\frac{\left(6 \pi^{4}\right)^{1 / 3} a_{4}}{960 M^{1 / 3} \gamma^{7 / 3}}\left(T-T_{c}\right)^{4 / 3}+O\left(T-T_{c}\right)^{5 / 3}, \\
P_{e r} & =\frac{2 \pi}{\sqrt{M}}+\frac{\pi(M-m) \sqrt{M}}{\gamma}\left(T-T_{c}\right)-\frac{6^{2 / 3} \pi^{5 / 3} M^{5 / 6} P_{5}}{20 \gamma^{8 / 3}}\left(T-T_{c}\right)^{5 / 3}+O\left(T-T_{c}\right)^{2}, \\
\mu_{r} & =-\frac{(48 \pi)^{1 / 3} M^{7 / 6} \gamma^{2 / 3}}{D^{2}+M^{3}}\left(T-T_{c}\right)^{1 / 3}+O\left(T-T_{c}\right)^{2 / 3}, \\
\mu_{a} & =\frac{2 \pi D}{M^{3 / 2}}+\frac{(48 \pi)^{1 / 3}}{M^{5 / 6}}\left(\frac{M t-D}{\gamma^{1 / 3}}+\frac{M^{2} \gamma^{2 / 3}}{D^{2}+M^{3}}\right)\left(T-T_{c}\right)^{1 / 3}+O\left(T-T_{c}\right)^{2 / 3},
\end{aligned}
$$

where

$$
\begin{aligned}
a_{4} & =-120 t M^{5}+\left(120 D+2 t^{3}+21 m t+72 d\right) M^{4} \\
& +\left[-\left(93 m+27 t^{2}\right) D+\left(27 m-2 t^{2}\right) d\right] M^{3}+\left(2 t^{2} m+25 d t-27 m^{2}\right) D M^{2} \\
& +\left[25 D^{3}+23(m t-d) D^{2}\right] M-25 m D^{3}, \\
P_{5} & =\left[M(M-m)^{2}+(M t-d)^{2}\right](M t-D) .
\end{aligned}
$$

In particular, if $\gamma>0$ and $D<0$, then the limit cycle bifurcates for $T>T_{c}$ and is orbitally asymptotically stable.

This theorem describes a codimension-one bifurcation, similar to the Hopf bifurcation of differentiable dynamics (see [CH82]), but some differences should be noted. In particular, the expressions characterizing the bifurcation are in terms of the parameter to the one third power instead of the one half power, and, more important, the limit cycle amplitude's leading order is $O(1)$. Thus, the stability change of the origin is accompanied by the abrupt appearance of a limit cycle of significant size. This comment also applies to the planar case, as appeared in [Kr87] and [FPR99].

When the coefficient $\gamma$ is not equal to zero, it allows a complete characterization of the bifurcation criticality. Its role is analogous to the coefficient of the cubic term in the Poincaré-Andronov-Hopf normal form. When $\gamma=0$, the bifurcation is of higher codimension, requiring a specific treatment that will appear elsewhere.

We want to remark that it is possible, with the same techniques, to obtain similar bifurcation results for the asymmetric case of single-sided saturation. Thus, the proposed methodology is able to cope with a wider class of piecewise linear systems.

The rest of the paper is structured as follows. In section 2, we show how the above result can be useful for accurately predicting the birth of symmetrical periodic 
oscillations in a tridimensional electronic circuit, which can be built by taking a Van der Pol oscillator as starting point. The proof of Theorem 1.1 is given in section 3 .

2. Predicting the onset of symmetrical periodic oscillations in a 3D electronic circuit. In this section, we consider the electronic circuit of Figure 2(a), genealogically related with the classical Van der Pol oscillator, in order to show the applicability of our results. Regarding this circuit, the nonlinear conductance NL is its active element, implemented by means of an operational amplifier with the feedback structure of Figure 2(b), and the current-voltage characteristic is shown in Figure 2 (c). Note that we are dealing with a nonlinearity characteristic qualitatively similar to the cubic one appearing in the classical Rayleigh-Van der Pol oscillator. In fact, if we eliminate the capacitor $C_{2}$ and make $R=R_{0}=0$, then the resulting planar circuit could be thought of as a modern electronic realization of such classical oscillators; see [Kr87] and [FPR99].

Thus, the 3D circuit of Figure 2 can be built by adding the capacitance $C_{2}$ to a bidimensional oscillator circuit. In the context of chaotic circuits, such topology was originally proposed in [SYM81], and was studied afterwards in [FGA84] and [FRGP93] in the case $R_{0}=0$ and assuming a nonlinear positive conductance for the resistor $R$. With slight modifications, this circuit has been extensively studied in the last two decades; see [GK92] or [HBCJM91]. Taking $R_{0}=0$ and substituting the nonlinear element by the so-called Chua diode, many papers have also been written; see [CWHZ93], [Ma93], and references therein. Anyway, the onset of symmetrical periodic oscillations was never accurately predicted, since in most cases the circuit was analyzed by taking polynomial approximations. Thus, the rapid bifurcation for the limit cycle observed in practice was never justified.

It should be remarked that the characteristic of Chua's diode is qualitatively similar to the one presented in Figure 2(c) but the zone of negative slope is made up by three pieces with two different slopes. For that, at least two subcircuits with operational amplifiers like those shown in Figure 2(b) are needed. Thus, the Chua circuit characteristic has five linear segments instead of only three, as in our case. However, in modeling Chua's circuit, usually only the three innermost pieces are represented, since the two outermost pieces of positive slope are not physically used; see [Ke93].

As stated in [Kr87] and [FPR99], there exists an excellent agreement between the actual response of the nonlinear device NL in the circuit and its symmetric piecewise

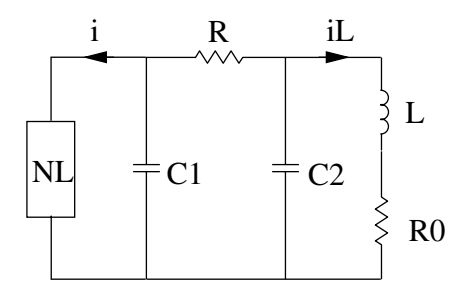

(a)

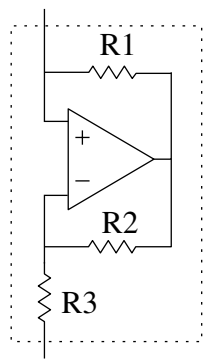

(b)

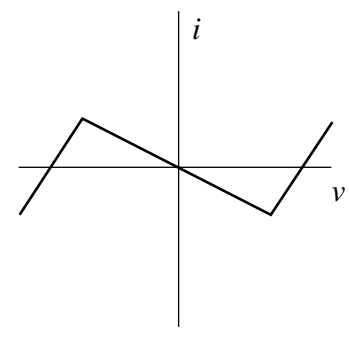

(c)

FIG. 2. (a) The $3 D$ electronic circuit. (b) Implementation of the nonlinear conductance NL. (c) Piecewise linear current-voltage characteristic of $N L$. 
linear mathematical model. Therefore, we are led to consider the piecewise linear dynamical system

$$
\begin{aligned}
C_{1} \frac{d v_{1}}{d \tau} & =\frac{v_{2}-v_{1}}{R}-i\left(v_{1}\right), \\
C_{2} \frac{d v_{2}}{d \tau} & =\frac{v_{1}-v_{2}}{R}-i_{L}, \\
L \frac{d i_{L}}{d \tau} & =v_{2}-R_{0} i_{L},
\end{aligned}
$$

where $v_{1}$ and $v_{2}$ are the voltages across the capacitors $C_{1}$ and $C_{2}$, respectively, while $i_{L}$ is the current through the inductance. The nonlinear current-voltage characteristic is

$$
i\left(v_{1}\right)=\frac{v_{1}-f\left(v_{1}\right)}{R_{1}} \quad \text { with } f\left(v_{1}\right)= \begin{cases}E \operatorname{sign}\left(v_{1}\right), & \left|v_{1}\right|>E / \sigma \\ \sigma v_{1}, & \left|v_{1}\right| \leq E / \sigma,\end{cases}
$$

where

$$
\sigma=1+\frac{R_{2}}{R_{3}}
$$

is the gain of the operational amplifier configured (using feedback) as a noninverting amplifier and $E$ is its saturation voltage.

With the following linear change of variables and time rescaling,

$$
v_{1}=\frac{E}{\sigma} y_{1}, \quad v_{2}=\frac{E}{\sigma} y_{2}, \quad i_{L}=\frac{E}{\sigma} \sqrt{\frac{C_{2}}{L}} y_{3}, \quad \tau=R C_{1} \bar{\tau},
$$

and defining the following five nonnegative dimensionless parameters,

$$
r=\frac{R}{R_{1}}, \quad c=\frac{C_{1}}{C_{2}}, \quad \mu=(\sigma-1) \frac{R}{R_{1}}=\frac{R R_{2}}{R_{1} R_{3}}, \quad \rho=\frac{R^{2} C_{1}^{2}}{L C_{2}}, \quad \kappa=\frac{R R_{0} C_{1}}{L},
$$

we can express system (2.1) as follows:

$$
\frac{d}{d \bar{\tau}}\left[\begin{array}{l}
y_{1} \\
y_{2} \\
y_{3}
\end{array}\right]=\left[\begin{array}{ccc}
-r-1 & 1 & 0 \\
c & -c & -\sqrt{\rho} \\
0 & \sqrt{\rho} & -\kappa
\end{array}\right]\left[\begin{array}{l}
y_{1} \\
y_{2} \\
y_{3}
\end{array}\right]+\left[\begin{array}{c}
\mu+r \\
0 \\
0
\end{array}\right] \operatorname{sat}\left(y_{1}\right) .
$$

For the subsequent analysis, we will choose $\mu$ and $\rho$ as the main bifurcation parameters. In practice, to detect the bifurcation in a experimental way, it is better to tune the parameter $\mu$ by means of a variable resistor $R_{2}$, which is equivalent to varying the gain $\sigma$.

The observability matrix for system $(2.1)$ is

$$
\mathcal{O}=\left[\begin{array}{c}
\mathbf{e}_{1}^{T} \\
\mathbf{e}_{1}^{T} A \\
\mathbf{e}_{1}^{T} A^{2}
\end{array}\right]=\left[\begin{array}{ccc}
1 & 0 & 0 \\
-r-1 & 1 & 0 \\
(r+1)^{2}+c & -c-r-1 & -\sqrt{\rho}
\end{array}\right],
$$

which has full rank for all the values of components of the circuit. From Proposition 16 of [CFPT02], system (2.1) can be expressed in Liénard's generalized form (1.2) with the following values:

$$
\begin{array}{ll}
T=\mu-c-\kappa-1, & t=-r-c-\kappa-1<0, \\
M=(c+1) \kappa-(c+\kappa) \mu+\rho, & m=(c+1) \kappa+(c+\kappa) r+\rho>0, \\
D=(c \kappa+\rho) \mu-\rho, & d=-(c \kappa+\rho) r-\rho<0 .
\end{array}
$$




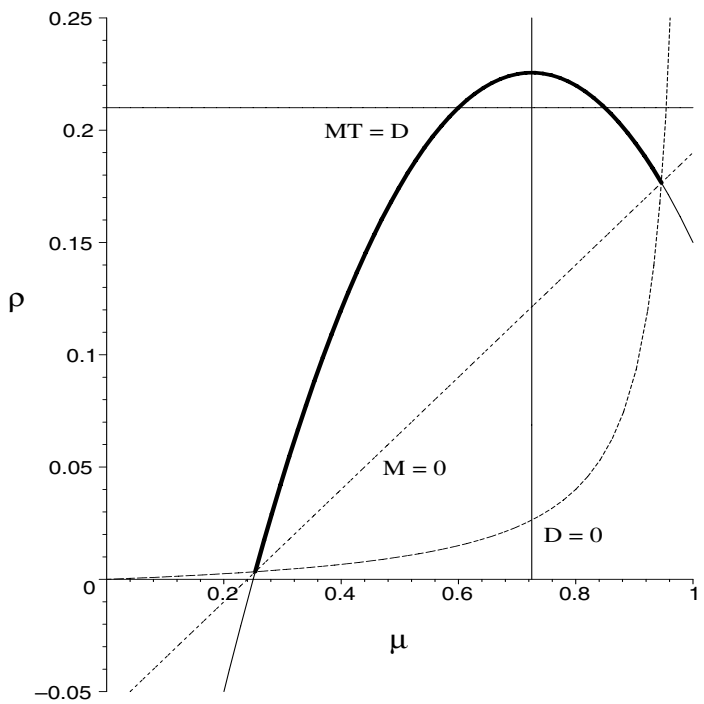

FIG. 3. The parabolic arc (thick line) in the plane $(\mu, \rho)$ corresponding to the bifurcation locus of Proposition 2.1 for $c=0.2$ and $\kappa=0.05$. The horizontal line indicates the path followed as $\mu$ varies for a fixed value of $\rho$. The dashed line represents points with $D=0$, so that above it we have $D<0$. At the dotted straight line we have $M=0$, and above this line we have $M>0$. The vertical line corresponds to $\mu=\mu_{*}$.

Note that these coefficients are the linear invariants of the two matrices involved, so that their computation is straightforward, and that it is not necessary to explicitly compute the linear change of variables required to get the Liénard form for applying Theorem 1.1.

The equation $M T-D=0$ leads to

$$
(c+\kappa) \mu^{2}-\left[(c+\kappa)^{2}+c+2 \kappa\right] \mu+\rho(c+\kappa)+\kappa(c+1)(c+\kappa+1)=0,
$$

which can be rewritten as $\left(\mu-\mu_{*}\right)^{2}+\rho-\rho_{*}=0$, where

$$
\begin{aligned}
& \mu_{*}=1+\frac{(c+\kappa)^{2}-c}{2(c+\kappa)} \\
& \rho_{*}=\mu_{*}^{2}-\kappa(c+1)\left(1+\frac{1}{c+\kappa}\right)
\end{aligned}
$$

represent the coordinates in the $(\mu, \rho)$-plane of the vertex of the quadratic (2.6); see Figure 3. Now the application of Theorem 1.1 allows us to state the following result.

Proposition 2.1. Let us consider system (2.4) and assume that $c>0$ and the parameter $\kappa$ satisfies

$$
0<\kappa<\kappa_{\max }(c)=\frac{\sqrt{c^{2}+c}-c}{2} .
$$

Then the system undergoes the focus-center-limit cycle bifurcation described in Theorem 1.1 at the points of the $(\mu, \rho)$-plane belonging to the parabolic arc defined by the quadratic equation

$$
\left(\mu-\mu_{*}\right)^{2}+\rho-\rho_{*}=0
$$




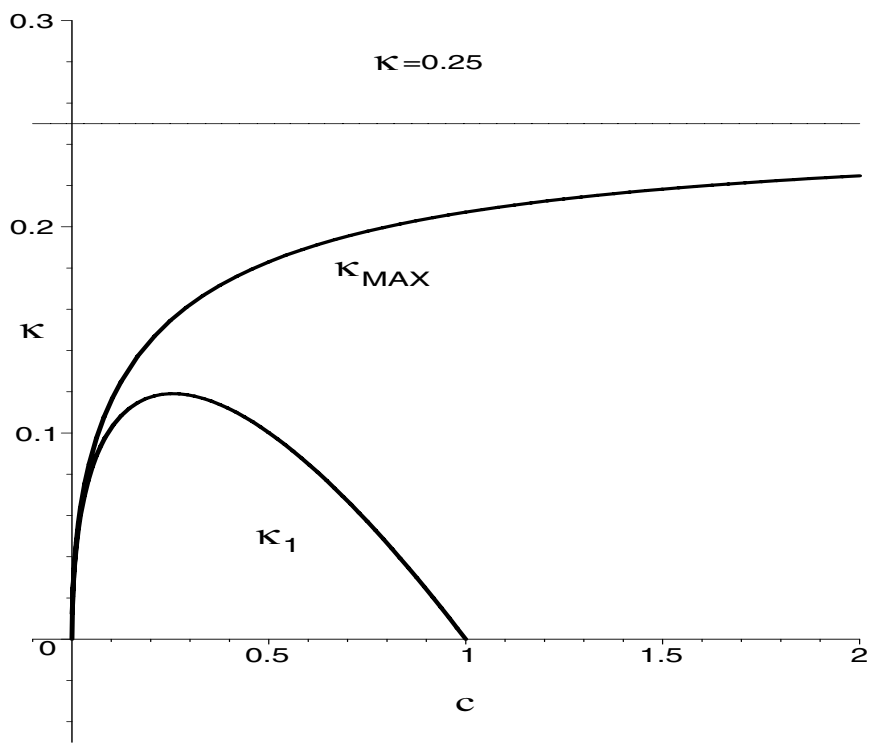

FIG. 4. The graphs of the functions $\kappa_{\max }(c)$ and $\kappa_{1}(c)$, which determine different regions in the plane $(c, \kappa)$ as described in statements (a) and (b) of Proposition 2.1. Note the horizontal asymptote at $\kappa=1 / 4$.

and satisfying

$$
\rho>(c+\kappa) \mu-(c+1) \kappa
$$

The endpoints of the above parabolic arc are

$\left(\mu_{1}, \rho_{1}\right)=\left(1-\frac{c+\underline{c}}{2(c+\kappa)}, \frac{c(1-2 \kappa)-\underline{c}}{2}\right),\left(\mu_{2}, \rho_{2}\right)=\left(1-\frac{c-\underline{c}}{2(c+\kappa)}, \frac{c(1-2 \kappa)+\underline{c}}{2}\right)$,

where

$$
\underline{c}=\sqrt{c^{2}(1-2 \kappa)^{2}-4 c(c+1) \kappa^{2}} .
$$

In the points of the above parabolic arc, the inequality $D<0$ holds, and the following cases arise:

(a) If $0<c<1$ and $0<\kappa<\kappa_{1}$, where $\kappa_{1}=\kappa_{1}(c)$ is the only positive root of the quartic

$$
(c+\kappa)^{4}+4 c \kappa(c+\kappa)-c^{2}=0
$$

then $\mu_{1}<\mu_{*}<\mu_{2}$ and two subcases appear; see Figure 4.

(a.1) If $\mu_{1}<\mu<\mu_{*}$, then at the bifurcation points of the parabolic arc given by (2.9)-(2.10) one has $\gamma>0$. Consequently, when $\rho$ varies, the bifurcation is supercritical and the limit cycle is orbitally asymptotically stable.

(a.2) If $\mu_{*}<\mu<\mu_{2}$, then $\gamma<0$ at the bifurcation points of the parabolic arc. Here, when $\rho$ varies, the bifurcation is subcritical and the limit cycle is unstable.

(b) If $0<c<1$ and $\kappa \geq \kappa_{1}$, or $c \geq 1$, then all the bifurcation points of the parabolic arc (2.9)-(2.10) satisfy $\gamma>0$. Therefore, the bifurcation is supercritical and the bifurcating limit cycle is orbitally asymptotically stable. 
TABLE 2.1

List of components for the circuit.

\begin{tabular}{|c|c|}
\hline$C=C_{1}=C_{2}$ & $100 \mathrm{nF}$ \\
\hline$L$ & $220 \mathrm{mH}$ \\
\hline$R_{1}$ & $10 \mathrm{k} \Omega$ \\
\hline$R_{3}$ & $2200 \Omega$ \\
\hline$R$ & $1 \mathrm{k} \Omega$ \\
\hline$R_{0}$ & $220 \Omega$ \\
\hline
\end{tabular}

Proof. Conditions $T=T_{c}$ and $M>0$ of Theorem 1.1 lead to $M T-D=0$, which is equivalent to (2.9), and to (2.10). After some manipulations, we get the inequality

$$
(c+\kappa) \mu^{2}-(c+2 \kappa) \mu+(c+1) \kappa<0,
$$

whose discriminant, namely $c^{2}-4 c^{2} \kappa-4 c \kappa^{2}$, is positive due to (2.8). In fact, this expression coincides with $\underline{c}^{2}$. The endpoints of the parabolic arc can be obtained by solving the equation $M=0$ and (2.9).

To show that $D<0$ at the bifurcation values, as we are working at points where $M T-D=0$ along with $M>0$, it suffices to show that $T<0$, which is a trivial task.

To prove statements (a) and (b), it is enough to study the sign of the coefficient $\gamma$ in Theorem 1. Using the condition $M T-D=0$, we have

$$
\gamma=M T(M-m)+M(d-t M)=M[T(M-m)+d-t M],
$$

and with $M>0$ we get $\operatorname{sign}(\gamma)=\operatorname{sign}[T(M-m)+d-t M]$. Thus, using (2.5), (2.6), and canceling a factor $r+\mu>0$, we conclude that

$$
\operatorname{sign}(\gamma)=\operatorname{sign}\left[(c+\kappa)^{2}+c+2 \kappa-2(c+\kappa) \mu\right]=\operatorname{sign}\left(\mu_{*}-\mu\right) .
$$

Assume now that $0<c<1$ and $0<\kappa<\kappa_{1}$. Thus, the left-hand side of (2.11) is negative, which implies $(c+\kappa)^{2}<\underline{c}$. Then $\mu_{1}<\mu_{*}<\mu_{2}$, and statement (a) follows.

When $c \leq 1$ and $\kappa \geq \kappa_{1}$, we have $(c+\kappa)^{2} \geq \underline{c}$. If $c>1$, then we have $(c+\kappa)^{2}>$ $c>\underline{c}$. In both cases, we conclude that $\mu_{*} \geq \mu_{2}$, and statement (b) follows.

For the sake of completeness, if we define, for $0<c<1$, the constants

$$
q_{1}=\sqrt[3]{27 c+26+3 \sqrt{81 c^{2}+156 c+75}}>0, \quad q_{2}=q_{1}+\frac{1}{q_{1}}-4>0,
$$

we obtain

$$
\kappa_{1}(c)=\sqrt{\frac{c}{6} q_{2}}+\sqrt{c \sqrt{\frac{6 c}{q_{2}}}-\frac{c q_{2}}{6}-2 c}-c,
$$

which is represented for $0<c<1$ in Figure 4 .

The above proposition enables us to design the electronic oscillator by choosing adequately the component values of the circuit. In particular, in order to minimize the signal distortion from the sinusoidal wave form, one must select parameters not far from the bifurcation curve where the onset of periodic oscillations has been predicted.

To assess the accuracy of piecewise linear modeling for this circuit, a SPICE implementation of the circuit was made; see [QNPS93]. The values chosen for the components are in Table 2.1, while the operational amplifier used was an LM324, 


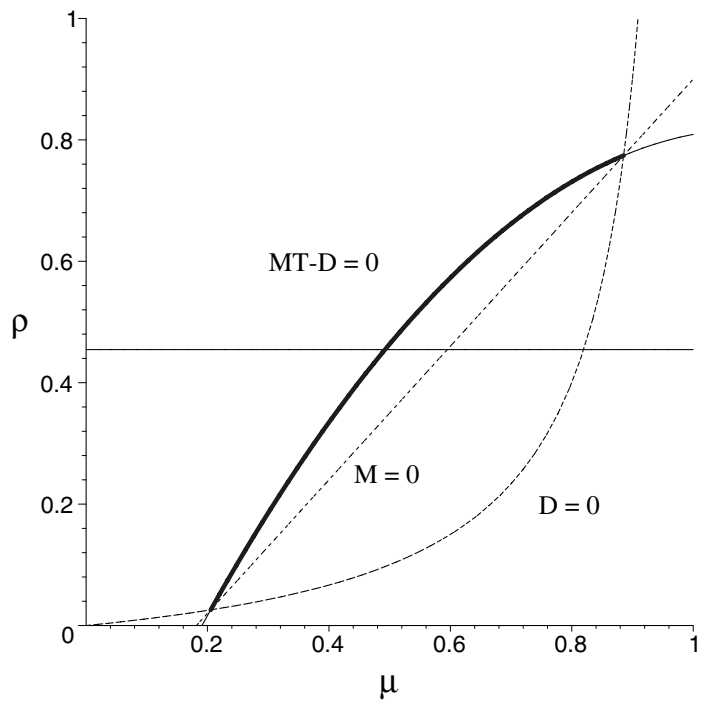

FIG. 5. The parabolic arc (thick line) in plane $(\mu, \rho)$ predicted by Proposition 2.1 for $\kappa=0.1$. The horizontal line indicates the path followed as $\mu$ varies for the fixed value of $\rho$ used in the simulations. The dashed line represents points with $D=0$, so that above it we have $D<0$. The dotted straight line indicates points with $M=0$, and above it we have $M>0$.

with a supply voltage of $9 \mathrm{~V}$ and a measured saturation voltage of $8.5 \mathrm{~V}$ (with slight variations around).

For these values, we have

$$
c=\frac{C_{1}}{C_{2}}=1, \quad \kappa=\frac{R R_{0} C}{L}=0.1<\frac{\sqrt{2}-1}{2} \approx 0.2071,
$$

so that we can apply Proposition 2.1 and, in particular, its statement (b). Note that

$$
\mu=\frac{R R_{2}}{R_{1} R_{3}} \approx \frac{R_{2}}{22000}, \quad \rho=\frac{R^{2} C}{L} \approx 0.4545,
$$

so that by varying $R_{2}$ we move $\mu$, describing a horizontal path that crosses the curve corresponding to the locus of bifurcation points, as shown in Figure 5. For the above value of $\rho$, the bifurcation takes place for the value $\bar{\mu} \approx 0.4924$, in accordance with (2.6), that corresponds with the value $R_{2} \approx 10833 \Omega$, and oscillations will appear by increasing $R_{2}$ above this critical value.

In Figures 6 and 7, we show the comparison between some experimental results taken from a SPICE simulation, once put into dimensionless form, and the predictions of Theorem 1.1 for the amplitude and the period of the bifurcating limit cycle. The excellent agreement achieved validates the piecewise linear model assumed for the operational amplifier nonlinear characteristic.

3. Proof of Theorem 1.1. In this section we provide the results necessary to prove Theorem 1.1.

For the critical value of the bifurcation parameter $T_{c}=D / M$, the matrix $A_{C}$ has a pair of imaginary eigenvalues, so that for $T$ in a neighborhood of $T_{c}$ the eigenvalues of $A_{C}$ will be $\alpha \pm i \beta$ and $\delta \in \mathbb{R}$. The characteristic polynomial of $A_{C}$ is

$$
p(\lambda)=\operatorname{det}\left(A_{C}-\lambda I\right)=-\lambda^{3}+T \lambda^{2}-M \lambda+D,
$$




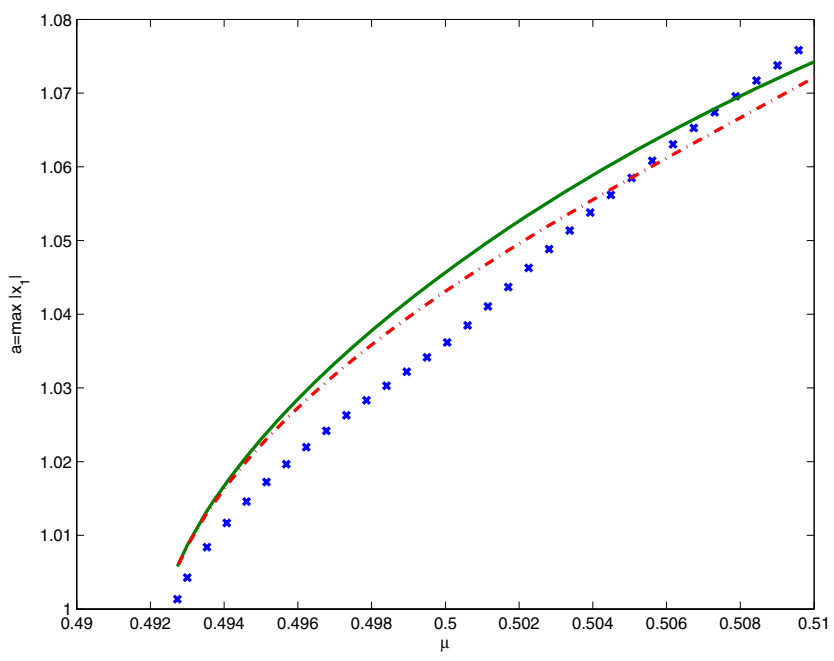

FIG. 6. Comparison for amplitude between SPICE simulation data $(\times \times \times)$, the expression corresponding to the two first non-null terms of Theorem 1.1 (-), and three non-null terms (---).

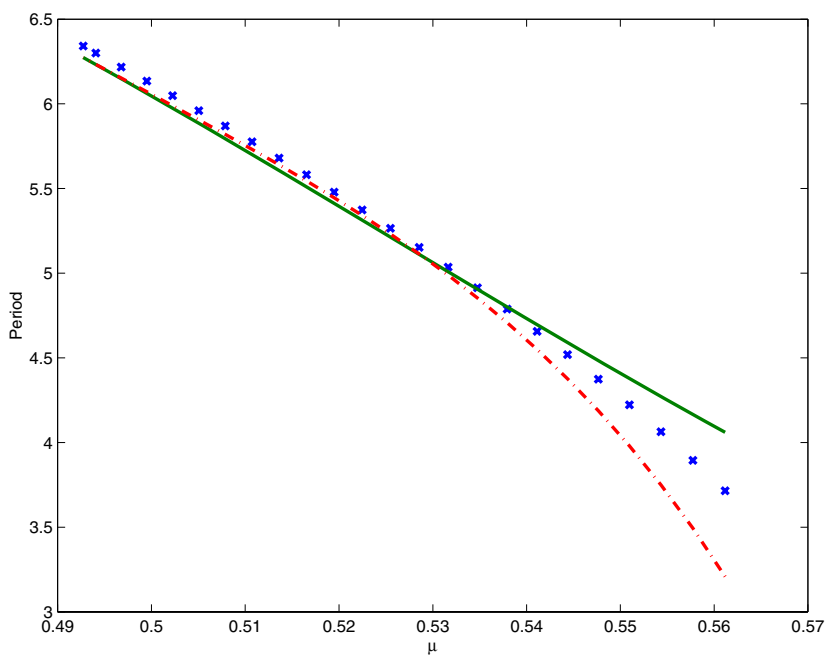

FIG. 7. Comparison for period between SPICE simulation data $(\times \times \times)$, the expression corresponding to the two first non-null terms of Theorem 1.1 (-), and three non-null terms (---).

and thus

$$
\begin{aligned}
T & =\delta+2 \alpha, \\
M & =2 \alpha \delta+\alpha^{2}+\beta^{2}, \\
D & =\delta\left(\alpha^{2}+\beta^{2}\right) .
\end{aligned}
$$

When $\alpha=0$ and $\beta>0$, or equivalently $D=M T$ and $M>0$, system (1.2) has a linear center contained in an invariant plane given by $\delta^{2} x_{1}-\delta x_{2}+x_{3}=0$. Additionally, the outermost periodic orbit of the center is tangent to the planes $\Sigma_{1}$ and $\Sigma_{-1}$ at the points $[1, \delta, 0]^{T}$ and $[-1,-\delta, 0]^{T}$, respectively. Consequently, the time 


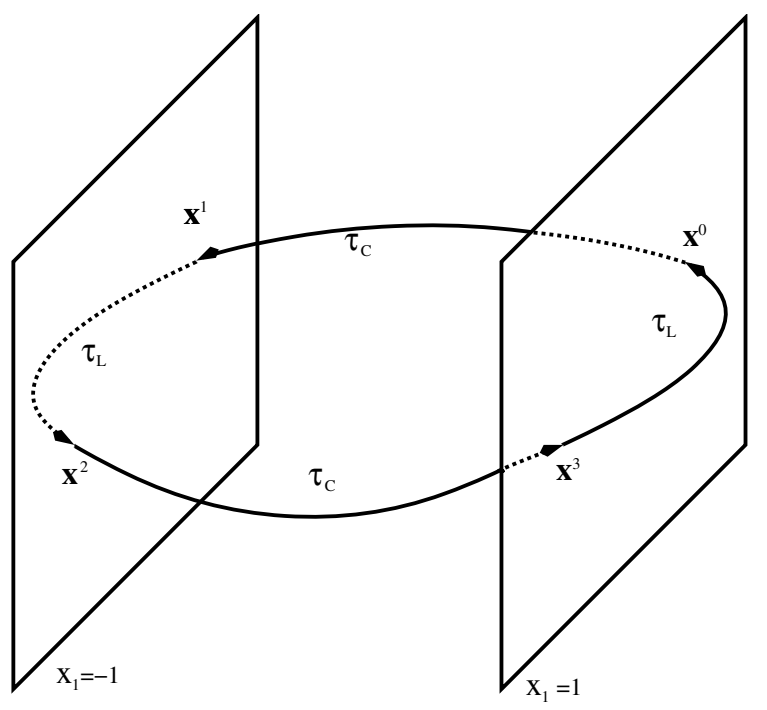

FIG. 8. Sketch of a symmetrical periodic orbit which uses the three linear zones of system (1.2).

spent by this orbit in going from $\mathbf{x}^{0}$ to $\mathbf{x}^{1}$ is $\tau_{C}=\pi / \beta$ in the zone $C$, and obviously $\tau_{L}=0$ in the zone $L$.

We want to analyze the possible bifurcation of a limit cycle from the linear center in the zone $C$. (Obviously, it should be born from the outermost periodic orbit of the center.) As system (1.2) is linear in every zone, it is possible to obtain its solutions explicitly, and to identify symmetrical periodic solutions of the system living in the three zones with the solutions of the equations

$$
\begin{aligned}
& e^{A_{C} \tau_{C}} \mathbf{x}^{0}-\mathbf{x}^{1}=\mathbf{0}, \\
& e^{A_{L} \tau_{L}} \mathbf{x}^{1}-\int_{0}^{\tau_{L}} e^{A_{L}\left(\tau_{L}-s\right)} \mathbf{b} d s+\mathbf{x}^{0}=\mathbf{0},
\end{aligned}
$$

where $\tau_{C}$ and $\tau_{L}$ are the times spent by the semiorbit in each zone, and

$$
\mathbf{x}^{0}=\left[\begin{array}{c}
1 \\
x_{2}^{0} \\
x_{3}^{0}
\end{array}\right], \quad \mathbf{x}^{1}=\left[\begin{array}{c}
-1 \\
x_{2}^{1} \\
x_{3}^{1}
\end{array}\right],
$$

are two intersection points of the orbit with the planes $\Sigma_{1}$ and $\Sigma_{-1}$, respectively (from the symmetry, there will be two more, $\mathbf{x}^{2}=-\mathbf{x}^{0}$ and $\mathbf{x}^{3}=-\mathbf{x}^{1}$ ); see Figure 8 . We will refer to the system formed by (3.2) as the closing equations. The use of these equations goes back to Andronov and coworkers [AVK66], and it was exploited by Kriegsmann [Kr87] in the context of limit cycle bifurcations. This author studied the rapid bifurcation in the Wien bridge oscillator, later revisited in [FPR99].

Starting from the critical value $T=T_{c}$ and considering the outermost periodic orbit of the corresponding center configuration, we want to use the closing equations to analyze what happens with such periodic orbit as $T$ varies, keeping $M$ and $D$ constant and always assuming $M>0$. To achieve this goal, it is more convenient to vary the eigenvalues of $A_{C}$ in a neighborhood of $(\alpha, \beta, \delta)=(0, \sqrt{M}, D / M)$, adding to the closing equations (3.2) the last two equations of (3.1), to impose that $M$ and $D$ are fixed. 

by

The sorted set formed by (3.2) and the last two equations of (3.1) will be denoted

$$
\mathbf{F}(\mathbf{z})=\mathbf{0}
$$

where $\mathbf{z}=\left(\alpha, \beta, \delta, \tau_{C}, \tau_{L}, x_{2}^{0}, x_{3}^{0}, x_{2}^{1}, x_{3}^{1}\right)$, which constitutes a nonlinear system of eight equations and nine unknowns, to be studied in a neighborhood of the point

$$
\overline{\mathbf{z}}=\left(0, \sqrt{M}, \frac{D}{M}, \frac{\pi}{\sqrt{M}}, 0, \frac{D}{M}, 0,-\frac{D}{M}, 0\right) .
$$

Obviously, we are interested in a branch of solutions of (3.3) passing through $\overline{\mathbf{z}}$, and leading to positive values of $\tau_{L}$. It turns out that system (3.3) has a trivial branch of solutions that passes through $\overline{\mathbf{z}}$ and can be parameterized as

$$
\mathbf{z}(\mu)=\left(0, \sqrt{M}, \frac{D}{M}, \frac{\pi}{\sqrt{M}}, 0, \frac{D}{M}+\mu, \mu \frac{D}{M},-\frac{D}{M}-\mu,-\mu \frac{D}{M}\right)
$$

for every real $\mu$. This trivial branch will be called the spurious branch because, for $\mu \neq 0$, these solutions do not correspond to periodic orbits of the system (1.2). The Jacobian matrix of $\mathbf{F}$ in $\overline{\mathbf{z}}$ does not have full rank; in fact, as the following result shows, the point $\overline{\mathbf{z}}$ is a branch point where two branches intersect each other. Moreover, we obtain a new set of equations for which $\overline{\mathbf{z}}$ is nonsingular.

LEMMA 3.1. For the closing equations (3.3) with $M>0$, the following statements hold:

(a) The fourth equation of (3.3), namely

$$
F_{4}(\mathbf{z})=0
$$

is satisfied for every $\mathbf{z}$ with $\tau_{L}=0$.

(b) The function $\widetilde{F}_{4}(\mathbf{z})$ such that $F_{4}(\mathbf{z})=\tau_{L} \widetilde{F}_{4}(\mathbf{z})$ is an analytic function in a neighborhood of $\overline{\mathbf{z}}$.

(c) If we define the modified closing equations

$$
\mathbf{G}(\mathbf{z})=\mathbf{0},
$$

where $G_{4}=\widetilde{F}_{4}$ and $G_{i}=F_{i}$ for $i \neq 4$, then the solution set of (3.5) in a neighborhood of $\bar{z}$ is the solution set of (3.3) excepting the spurious branch (3.4).

(d) For system (3.5) the point $\bar{z}$ is a nonsingular point. Moreover, the solutions of (3.5) are analytic functions of $\tau_{L}$ at 0 , and their corresponding Taylor series are

$$
\begin{aligned}
\alpha & =\frac{M^{5 / 2} \gamma}{12 \pi\left(D^{2}+M^{3}\right)} \tau_{L}^{3}+\frac{M^{1 / 2} \xi_{1}}{720 p i\left(D^{2}+M^{3}\right)} \tau_{L}^{5}+O\left(\tau_{L}^{6}\right), \\
\beta & =\sqrt{M}-\frac{D M \gamma}{12 \pi\left(D^{2}+M^{3}\right)} \tau_{L}^{3}-\frac{D \xi_{1}}{720 \pi M\left(D^{2}+M^{3}\right)} \tau_{L}^{5}+O\left(\tau_{L}^{6}\right), \\
\delta & =\frac{D}{M}+\frac{D^{2} \gamma}{6 \pi M^{1 / 2}\left(D^{2}+M^{3}\right)} \tau_{L}^{3}+\frac{D^{2} \xi_{1}}{360 \pi M^{5 / 2}\left(D^{2}+M^{3}\right)} \tau_{L}^{5}+O\left(\tau_{L}^{6}\right), \\
\tau_{C} & =\frac{\pi}{\sqrt{M}}-\tau_{L}+\frac{M-m}{12} \tau_{L}^{3}+\frac{\xi_{2}}{720 \pi M^{2}} \tau_{L}^{5}+O\left(\tau_{L}^{6}\right),
\end{aligned}
$$




$$
\begin{aligned}
x_{2}^{0} & =\frac{D}{M}+\frac{M}{2} \tau_{L}+\frac{M t-D}{12} \tau_{L}^{2}+\frac{4 M \gamma+\pi \sqrt{M} \xi_{3}}{24 \pi M^{3 / 2}} \tau_{L}^{3}-\frac{\xi_{4}}{720 M^{2}} \tau_{L}^{4} \\
& +\left[\frac{D \xi_{3}-M^{3} d}{24 M^{2}\left(e^{\pi D / M^{3 / 2}}+1\right)}-\frac{D M^{3 / 2} \gamma}{12 \pi\left(D^{2}+M^{3}\right)}\right] \tau_{L}^{4}+O\left(\tau_{L}^{5}\right), \\
x_{2}^{1} & =-\frac{D}{M}+\frac{M}{2} \tau_{L}-\frac{M t-D}{12} \tau_{L}^{2}-\frac{4 M \gamma-\pi \sqrt{M} \xi_{3}}{24 \pi M^{3 / 2}} \tau_{L}^{3}+\frac{\xi_{4}}{720 M^{2}} \tau_{L}^{4} \\
& -\left[\frac{e^{\pi D / M^{3 / 2}}\left(D \xi_{3}-M^{3} d\right)}{24 M^{2}\left(e^{\pi D / M^{3 / 2}}+1\right)}+\frac{D M^{3 / 2} \gamma}{12 \pi\left(D^{2}+M^{3}\right)}\right] \tau_{L}^{4}+O\left(\tau_{L}^{5}\right), \\
x_{3}^{0} & =\frac{D}{2} \tau_{L}+\frac{(M t-D) D}{12 M} \tau_{L}^{2}+\left[\frac{D M^{3 / 2} \gamma}{6 \pi\left(D^{2}+M^{3}\right)}+\frac{D \xi_{3}}{24 M^{2}}\right] \tau_{L}^{3} \\
& -\frac{D \xi_{3}-M^{3} d}{12 M^{2}\left(e^{\pi D / M^{3 / 2}}+1\right)} \tau_{L}^{3}-\frac{D \xi_{4}}{720 M^{3}} \tau_{L}^{4}+O\left(\tau_{L}^{5}\right), \\
x_{3}^{1} & =\frac{D}{2} \tau_{L}-\frac{(M t-D) D}{12 M} \tau_{L}^{2}-\left[\frac{D M^{3 / 2} \gamma}{6 \pi\left(D^{2}+M^{3}\right)}+\frac{D \xi_{3}}{24 M^{2}}\right] \tau_{L}^{3} \\
& +\frac{D \xi_{3}+e^{\pi D / M^{3 / 2}} M^{3} d}{12 M^{2}\left(e^{\pi D / M^{3 / 2}}+1\right)} \tau_{L}^{3}+\frac{D \xi_{4}}{720 M^{3}} \tau_{L}^{4}+O\left(\tau_{L}^{5}\right),
\end{aligned}
$$

where

$$
\begin{aligned}
\gamma & =D M-D m+d M-t M^{2}, \\
\xi_{1} & =5 D^{3} M-5 D^{3} m-15 D^{2} M^{2} t+11 D^{2} M d+4 D^{2} M m t-15 D M^{4} \\
& +21 D M^{3} m+9 D M^{3} t^{2}-10 D M^{2} d t-6 D M^{2} m^{2}+D M^{2} m t^{2}+15 M^{5} t \\
& -9 M^{4} d-12 M^{4} m t+M^{4} t^{3}+6 M^{3} d m-M^{3} d t^{2}, \\
\xi_{2} & =5 D^{2} M-5 D^{2} m-10 D M^{2} t+6 D M d+4 D M m t-9 M^{4} \\
& +15 M^{3} m+5 M^{3} t^{2}-6 M^{2} d t-6 M^{2} m^{2}+M^{2} m t^{2}, \\
\xi_{3} & =D(D-M t)+M^{2} m, \\
\xi_{4} & =15 D^{3}-20 D^{2} M t+16 D M^{2} m+4 D M^{2} t^{2}-9 M^{3} d-7 M^{3} m t+M^{3} t^{3} .
\end{aligned}
$$

Proof. Statements (a) and (b) come from a direct inspection of (3.3).

Recalling (3.2), from statement (b) and (3.3) we conclude that

$$
\left.\widetilde{F}_{4}(\mathbf{z})\right|_{\tau_{L}=0}=\lim _{\tau_{L} \rightarrow 0} \frac{1}{\tau_{L}} F_{4}(\mathbf{z})=\mathbf{e}_{1}^{T} A_{L}\left[\begin{array}{c}
-1 \\
x_{2}^{1} \\
x_{3}^{1}
\end{array}\right]+\mathbf{e}_{1}^{T}\left[\begin{array}{c}
t-2 \alpha-\delta \\
m-M \\
d-D
\end{array}\right]=-x_{2}^{1}-2 \alpha-\delta .
$$

The above computation shows that $\widetilde{F}_{4}(\mathbf{z}(\mu))=\mu$, so that the spurious branch (3.4) does not belong to the solution set of $\widetilde{F}_{4}(\mathbf{z})=0$. Besides, every solution $\mathbf{z}$ of (3.3) with $\tau_{L} \neq 0$ is a solution of (3.5), and statement (c) is proven.

For the computation of the Jacobian matrix $\partial \mathbf{G} /\left.\partial \mathbf{z}\right|_{\mathbf{z}=\overline{\mathbf{z}}}$ and the series (3.6)(3.13), we have used the following approach. For the first three rows of the closing equations, we work with the equivalent expression

$$
\mu_{0} e^{\delta \tau_{C}} \mathbf{v}+e^{\alpha \tau_{C}} \cos \left(\beta \tau_{C}\right) \widehat{\mathbf{v}}+e^{\alpha \tau_{C}} \frac{\sin \left(\beta \tau_{C}\right)}{\beta}\left(A_{C}-\alpha I\right) \widehat{\mathbf{v}}-\mathbf{x}^{1}=\mathbf{0},
$$


where $\mathbf{v}=\left[1,2 \alpha, \alpha^{2}+\beta^{2}\right]^{T}$ is a right eigenvector of $A_{C}$ associated with the real eigenvalue $\delta$, and

$$
\widehat{\mathbf{v}}=\mathbf{x}^{0}-\mu_{0} \mathbf{v}
$$

is the projection (following the direction of $\mathbf{v}$ ) of the vector $\mathbf{x}^{0}$ onto the invariant plane associated with the complex eigenvalues of $A_{C}$. Consequently, the coefficient $\mu_{0}$ is

$$
\mu_{0}=\frac{\mathbf{w}^{T} \mathbf{x}^{0}}{\mathbf{w}^{T} \mathbf{v}}=\frac{\delta^{2}-\delta x_{2}^{0}+x_{3}^{0}}{(\delta-\alpha)^{2}+\beta^{2}}
$$

where $\mathbf{w}^{T}=\left[\delta^{2},-\delta, 1\right]$ is a left eigenvector of $A_{C}$ associated with the eigenvalue $\delta$.

Regarding the next three rows of the closing equations, it is useful to write the matrix exponentials in series of $\tau_{L}$. Then, in computing partial derivatives with respect to the variables other from $\tau_{L}$, one only needs to consider the terms of degree zero in $\tau_{L}$. This comment is also useful for obtaining $\widetilde{F}_{4}$ from $F_{4}$.

Thus, the Jacobian matrix $\partial \mathbf{G} /\left.\partial \mathbf{z}\right|_{\mathbf{z}=\overline{\mathbf{z}}}$ is

$$
\left[\begin{array}{ccccccccc}
-\frac{\pi}{\sqrt{M}} & 0 & D M K & 0 & 0 & -D M K & M^{2} K & 0 & 0 \\
-\frac{\pi D}{M^{3 / 2}} & -\pi & 0 & -M & 0 & -1 & 0 & -1 & 0 \\
0 & -\frac{\pi D}{M} & D M^{2} K & -D & 0 & -D M^{2} K & M^{3} K-1 & 0 & -1 \\
-2 & 0 & -1 & 0 & \frac{M}{2} & 0 & 0 & -1 & 0 \\
0 & 0 & 0 & 0 & -M & 1 & 0 & 1 & 0 \\
0 & 0 & 0 & 0 & -D & 0 & 1 & 0 & 1 \\
\frac{2 D}{M} & 2 \sqrt{M} & 0 & 0 & 0 & 0 & 0 & 0 & 0 \\
0 & \frac{2 D}{\sqrt{M}} & M & 0 & 0 & 0 & 0 & 0 & 0
\end{array}\right],
$$

where

$$
K=\frac{e^{\pi D / M^{3 / 2}}+1}{D^{2}+M^{3}} .
$$

If we remove the fifth column (corresponding to $\tau_{L}$ ), the determinant of the resulting matrix is equal to

$$
-2 \pi M^{2}\left(e^{\pi D / M^{3 / 2}}+1\right) \neq 0,
$$

and hence the matrix has full rank. From the implicit function theorem for analytic functions (see [CH82]) we obtain statement (d). All the computations of the above series expansions have been checked with the symbolic manipulator Maple; see [MGHLVM03].

In what follows, we give an auxiliary result to analyze the stability of the bifurcating limit cycle. First, we must study the behavior of the return map near a periodic orbit of three zones. Due to the symmetry, we need to use only the semiorbit that starts from $\mathbf{x}^{0} \in \Sigma_{1}$, crosses $\Sigma_{-1}$ at the point $\mathbf{x}^{1}$, and returns to this section at the point $\mathbf{x}^{2}=-\mathbf{x}^{0} \in \Sigma_{-1}$. We denote by $\mathbf{p}_{0}, \mathbf{p}_{1} \in \mathbb{R}^{2}$, the coordinates of $\mathbf{x}^{0}$ and $\mathbf{x}^{1}$ restricted to their respective sections. From the transition maps associated with the flow, locally defined at the points $\mathbf{x}^{0}$ and $\mathbf{x}^{1}$, it is possible in adequate neighborhoods at the sections to define the functions providing the corresponding restricted coordinates. Let us denote by $\pi_{C}, \pi_{L}$ such functions, satisfying $\pi_{C}\left(\mathbf{p}_{0}\right)=\mathbf{p}_{1}, \pi_{L}\left(\mathbf{p}_{1}\right)=-\mathbf{p}_{0}$, 
and let $\pi_{L C}=\pi_{L} \circ \pi_{C}$. We will let $\tau_{C}\left(\mathbf{p}_{0}\right)$ and $\tau_{L}\left(\mathbf{p}_{1}\right)$ denote the times spent by the semiorbit in passing from $\mathbf{x}^{0}$ to $\mathbf{x}^{1}$ and from $\mathbf{x}^{1}$ to $\mathbf{x}^{2}$, respectively, and write $D_{\mathbf{p}}(\cdot)$ to indicate the derivative with respect to the restricted coordinates. The next result shows how to compute the derivative $D_{\mathbf{p}} \pi_{L C}$, intimately related to the monodromy matrix associated with the periodic orbit.

Proposition 3.2. Consider a symmetrical periodic orbit of system (1.2) that uses the three zones, starting from $\mathbf{x}^{0} \in \Sigma_{1}$ with coordinates $\mathbf{p}_{0} \in \mathbb{R}^{2}$, passing through $\mathbf{x}^{1} \in \Sigma_{-1}$ with coordinates $\mathbf{p}_{1} \in \mathbb{R}^{2}$, and transversal to both sections. Then, the product of the two matrices

$$
\left[\begin{array}{ll}
1 & D_{\mathbf{p}} \tau_{L}\left(\mathbf{p}_{1}\right) \\
\mathbf{0} & D_{\mathbf{p}} \pi_{L}\left(\mathbf{p}_{1}\right)
\end{array}\right]\left[\begin{array}{cc}
-1 & D_{\mathbf{p}} \tau_{C}\left(\mathbf{p}_{0}\right) \\
\mathbf{0} & D_{\mathbf{p}} \pi_{C}\left(\mathbf{p}_{0}\right)
\end{array}\right]=\left[\begin{array}{cc}
-1 & D_{\mathbf{p}} \tau_{C}\left(\mathbf{p}_{0}\right)+D_{\mathbf{p}} \tau_{L}\left(\mathbf{p}_{1}\right) D_{\mathbf{p}} \pi_{C}\left(\mathbf{p}_{0}\right) \\
\mathbf{0} & D_{\mathbf{p}} \pi_{L C}\left(\mathbf{p}_{0}\right)
\end{array}\right]
$$

is similar to

$$
e^{A_{L} \tau_{L}\left(\mathbf{p}_{1}\right)} e^{A_{C} \tau_{C}\left(\mathbf{p}_{0}\right)} .
$$

Proof. It is enough to use the explicit expressions of the solutions of system (1.2) at every zone and the continuity of the vector field; see [Ro03] for more details.

The following lemma deals with a technical result that allows us to invert certain power series; see [FPR99] for a proof.

LEMMA 3.3. Let be $\eta=\xi^{n} \rho(\xi)$ with $n$ odd, where $\rho$ is a real function analytic at 0 and such that $\rho(0)=\rho_{0} \neq 0$. Then there exists a real function $\chi$ analytic at 0 , with $\chi(0) \neq 0$ and such that $\xi=\eta^{\frac{1}{n}} \chi\left(\eta^{\frac{1}{n}}\right)$.

If we select only the solutions of the closing equations with $\tau_{L}>0$ but sufficiently small, and $0<\tau_{C}<\pi / \sqrt{M}$ but sufficiently close to $\pi / \sqrt{M}$, then we can assure that such solutions correspond to symmetrical and transversal periodic orbits; see [Ro03] for more details. Reciprocally, if we take a symmetrical periodic orbit that uses the three zones and is sufficiently close to the outermost periodic orbit of the center that exists for the critical values of parameters, then its corresponding values $\tau_{C}>0$, $\tau_{L}>0, \mathbf{x}^{0}, \mathbf{x}^{1}$, and remaining parameters determine a point $\mathbf{z}$ satisfying the closing equations. Therefore, we can establish with the above restrictions a correspondence between solutions $\mathbf{z}$ of closing equations and symmetrical periodic orbits. This correspondence, along with the uniqueness of the solution obtained in Lemma 3.1, ensures that the corresponding bifurcating periodic is an isolated periodic orbit, that is, a limit cycle.

Coming back to the statements of Theorem 1.1, we begin by using statement (d) of Lemma 3.1. We can compute $T\left(\tau_{L}\right)$ using that $T=2 \alpha+\delta$ and the corresponding expansions (3.6) and (3.8) for $\alpha$ and $\delta$, obtaining

$$
T=\frac{D}{M}+\frac{\gamma}{6 \pi M^{1 / 2}} \tau_{L}^{3}+\frac{\xi_{1}}{360 \pi M^{5 / 2}} \tau_{L}^{5}+O\left(\tau_{L}^{6}\right)
$$

where $\gamma$ and $\xi_{1}$ are given in the statement of Lemma 3.1. From (3.17) and taking into consideration that $\tau_{L}$ must be positive, it is obvious that $M T-D$ and $\gamma$ have the same sign, and so the condition $\gamma(M T-D)>0$ holds.

Now, if we apply Lemma 3.3 to (3.17), taking $n=3, \eta=M T-D$, and $\xi=\tau_{L}$, we conclude that $\tau_{L}$ is an analytic function at the origin in the variable $(M T-D)^{1 / 3}$. A standard computation leads to the expansion

$$
\tau_{L}=\frac{(6 \pi)^{1 / 3}(M T-D)^{1 / 3}}{M^{1 / 6} \gamma^{1 / 3}}+\frac{\pi \xi_{1}}{30 M^{5 / 2} \gamma^{2}}(M T-D)+O(M T-D)^{4 / 3} .
$$


Due to the symmetry of the orbit, its period is equal to $2\left(\tau_{C}+\tau_{L}\right)$. Substituting expansion (3.18) into (3.9), and computing the above expression for the period, we get the expansion given for $P_{e r}$.

We will now determine the amplitude of the periodic orbit. By using the variation of parameters formula, the solution of system (1.2) in zone $R$ is

$$
\mathbf{x}(\tau)=e^{A_{L} \tau} \mathbf{x}^{3}\left(\tau_{L}\right)+\int_{0}^{\tau} e^{A_{L}(\tau-s)} \mathbf{b}\left(\tau_{L}\right) d s,
$$

so that its first component is

$$
x_{1}(\tau)=\mathbf{e}_{1}^{T}\left\{e^{A_{L} \tau}\left[\begin{array}{c}
1 \\
-x_{2}^{1}\left(\tau_{L}\right) \\
-x_{3}^{1}\left(\tau_{L}\right)
\end{array}\right]+\left(\sum_{i=0}^{\infty} A_{L}^{i} \frac{\tau^{i+1}}{(i+1) !}\right) \mathbf{b}\left(\tau_{L}\right)\right\} .
$$

Let $\tau^{*}$ be the time when $\left|x_{1}\right|$ attains its maximum value in zone $R$. Taking derivatives with respect to $\tau$ in (3.20), and imposing that it must vanish at $\tau^{*}$, we get

$$
G\left(\tau_{L}, \tau^{*}\right)=\left.\frac{d x_{1}(\tau)}{d \tau}\right|_{\tau=\tau^{*}}=\mathbf{e}_{1}^{T} e^{A_{L} \tau^{*}}\left[\begin{array}{c}
x_{2}^{1}\left(\tau_{L}\right)+T\left(\tau_{L}\right) \\
x_{3}^{1}\left(\tau_{L}\right)+M \\
D
\end{array}\right]=0 .
$$

Now using expressions (3.11) and (3.13) and computing the power series of $G$ in $\left(\tau_{L}, \tau^{*}\right)$ at $(0,0)$, we obtain

$$
G\left(\tau_{L}, \tau^{*}\right)=\frac{M}{2} \tau_{L}-M \tau^{*}+\frac{D-M t}{12} \tau_{L}^{2}+\frac{M t-D}{2} \tau_{L} \tau^{*}+\frac{D-M t}{2} \tau^{* 2}+O\left(\tau_{L}, \tau^{*}\right)^{3} .
$$

Hence, (3.21) defines implicitly in a neighborhood of $(0,0)$ a function $\tau^{*}=\psi\left(\tau_{L}\right)$ such that $G\left(\tau_{L}, \psi\left(\tau_{L}\right)\right)=0$, namely,

$$
\tau^{*}=\frac{1}{2} \tau_{L}+\frac{M t-D}{24 M} \tau_{L}^{2}+O\left(\tau_{L}^{4}\right)
$$

Substituting the above expansion together with (3.11), (3.13), and (3.17) into the expression (3.20), we get

$$
a=x_{1}\left(\tau^{*}\right)=1+\frac{M}{8} \tau_{L}^{2}+\frac{1}{1152 M}\left(13 D^{2}-11 D M t+15 M^{2} m-2 M^{2} t^{2}\right) \tau_{L}^{4}+O\left(\tau_{L}^{5}\right) .
$$

Using expression (3.18) for $\tau_{L}$, we obtain the final expression for the amplitude $a$.

Let us now compute the characteristic multipliers of the bifurcating limit cycle. Due to the similarity relationship established in Proposition 3.2, we conclude that the product $\exp \left(A_{L} \tau_{L}\right) \cdot \exp \left(A_{C} \tau_{C}\right)$ corresponding to a solution of (3.5) has an eigenvalue equal to -1 . We will denote by $\lambda_{r}$ and $\lambda_{a}$ the other two eigenvalues that correspond to the eigenvalues of the derivative $D_{\mathbf{p}} \pi_{L C}\left(\mathbf{p}_{0}\right)$ of the transition map associated with the semiorbit. The product of the three eigenvalues is then equal to

$$
-\lambda_{r} \lambda_{a}=\operatorname{det}\left(e^{A_{L} \tau_{L}}\right) \operatorname{det}\left(e^{A_{C} \tau_{C}}\right) .
$$

Using that $\operatorname{det}\left(e^{A \tau}\right)=\exp (\tau \operatorname{trace}(A))$, we get

$$
-\lambda_{r} \lambda_{a}=e^{\tau_{L} t+\tau_{C} T} .
$$


The expansion of the product of exponentials in (3.16) leads to an expression of the form

$$
e^{A_{L} \tau_{L}} e^{A_{C}\left(\tau_{L}\right) \tau_{C}\left(\tau_{L}\right)}=H_{0}+\tau_{L} H_{1}+\tau_{L}^{2} H_{2}+\cdots .
$$

To compute the above matrices $H_{i}$, we write

$$
\begin{aligned}
& \left(I+A_{L} \tau_{L}+A_{L}^{2} \frac{\tau_{L}^{2}}{2 !}+\cdots\right) \\
& \times\left(e^{A_{C}(0) \tau_{C}(0)}+\left.\tau_{L} \frac{d}{d \tau_{L}} e^{A_{C}\left(\tau_{L}\right) \tau_{C}\left(\tau_{L}\right)}\right|_{\tau_{L}=0}+\left.\frac{\tau_{L}^{2}}{2 !} \frac{d^{2}}{d \tau_{L}^{2}} e^{A_{C}\left(\tau_{L}\right) \tau_{C}\left(\tau_{L}\right)}\right|_{\tau_{L}=0}+\cdots\right) .
\end{aligned}
$$

From expansions (3.6)-(3.13), we obtain $\tau_{C}(0)=\pi / M^{1 / 2}, \tau_{C}^{\prime}(0)=-1, \tau_{C}^{\prime \prime}(0)=0$, $A_{C}^{\prime}(0)=A_{C}^{\prime \prime}(0)=\mathbf{0}$, and using these values in the above expression, we finally get

$$
\begin{gathered}
H_{0}=e^{A_{C}(0) \tau_{C}(0)}=\left[\begin{array}{ccc}
D^{2} K-1 & -D M K & M^{2} K \\
0 & -1 & 0 \\
D^{2} M K & -D M^{2} K & M^{3} K-1
\end{array}\right], \\
H_{1}=\left[\begin{array}{c}
t-D / M \\
m-M \\
d-D
\end{array}\right]\left[\begin{array}{lll}
D^{2} K-1 & -D M K & M^{2} K
\end{array}\right]
\end{gathered}
$$

and

$$
H_{2}=\frac{M t-D}{2 M} H_{1}
$$

where $K$ has been defined in (3.15), and it is emphasized that $H_{1}$ and $H_{2}$ are rank-one matrices.

The matrix $H_{0}$ has eigenvalues -1 (double) and $\lambda_{0}=\exp \left(\pi D / M^{3 / 2}\right)$. For the single eigenvalue $\lambda_{0}$, we select a right eigenvector $\mathbf{v}_{0}=[1,0, M]^{T}$ and a left eigenvector $\mathbf{w}_{0}^{T}=\left[D^{2} / M^{2},-D / M, 1\right]$. We will denote by $\lambda_{a}$ the eigenvalue of $D \pi_{L C}\left(\mathbf{p}_{0}\right)$ that for $\tau_{L}=0$ is equal to $\lambda_{0}$. Since the eigenvalue $\lambda_{0}$ of $H_{0}$ is simple, we can apply perturbation theory (see section 2.8 of [Wi65]) to assure that the equality

$$
\begin{aligned}
& \left(H_{0}+\tau_{L} H_{1}+\tau_{L}^{2} H_{2}+\cdots\right)\left(\mathbf{v}_{0}+\tau_{L} \mathbf{v}_{1}+\tau_{L}^{2} \mathbf{v}_{2}+\cdots\right) \\
& =\left(\lambda_{0}+\tau_{L} \lambda_{1}+\tau_{L}^{2} \lambda_{2}+\cdots\right)\left(\mathbf{v}_{0}+\tau_{L} \mathbf{v}_{1}+\tau_{L}^{2} \mathbf{v}_{2}+\cdots\right)
\end{aligned}
$$

holds for certain vectors $\mathbf{v}_{1}, \mathbf{v}_{2} \ldots$. As a consequence of Proposition 3.2 and (3.23), we get

$$
\lambda_{a}=\lambda_{0}+\tau_{L} \lambda_{1}+\tau_{L}^{2} \lambda_{2}+\cdots
$$

After some computations, we arrive at

$$
\begin{aligned}
\lambda_{1} & =\frac{\mathbf{w}_{0}^{T} H_{1} \mathbf{v}_{0}}{\mathbf{w}_{0}^{T} \mathbf{v}_{0}}=\left(\frac{M t-D}{M}+\frac{\gamma M}{D^{2}+M^{3}}\right) \lambda_{0}, \\
\lambda_{2} & =\frac{\mathbf{w}_{0}^{T}\left(H_{2} \mathbf{v}_{0}+\left(H_{1}-\lambda_{1} I\right) \mathbf{v}_{1}\right)}{\mathbf{w}_{0}^{T} \mathbf{v}_{0}}=\frac{(M t-D)\left(D^{2}+M^{3}\right)+\gamma}{2 M^{2}\left(D^{2}+M^{3}\right)^{2}\left(\lambda_{0}+1\right)} \\
& \times\left[(M t-D)\left[\left(D^{2}+M^{3}\right) \lambda_{0}+D^{2}-M^{3}\right]+2 M^{2}(d M-D m)\right] \lambda_{0} .
\end{aligned}
$$


The logarithms $\mu_{r}$ and $\mu_{a}$ of characteristic multipliers of the complete periodic orbit must satisfy

$$
e^{\mu_{r}}=\lambda_{r}^{2}, \quad e^{\mu_{a}}=\lambda_{a}^{2},
$$

while from (3.22) we get the relationship

$$
\mu_{r}+\mu_{a}=2 t \tau_{L}+2 T \tau_{C} .
$$

From (3.24) and using the computed simple eigenvalue $\lambda_{a}$, we obtain

$$
\begin{aligned}
\mu_{a} & =2 \log \left[\lambda_{0}+\lambda_{1} \tau_{L}+\lambda_{2} \tau_{L}^{2}+O\left(\tau_{L}^{3}\right)\right]=2 \log \lambda_{0}+2 \log \left[1+\frac{\lambda_{1}}{\lambda_{0}} \tau_{L}+\frac{\lambda_{2}}{\lambda_{0}} \tau_{L}^{2}+O\left(\tau_{L}^{3}\right)\right] \\
& =2 \lambda_{0}+2 \frac{\lambda_{1}}{\lambda_{0}} \tau_{L}+\left[2 \frac{\lambda_{2}}{\lambda_{0}}-\frac{\lambda_{1}^{2}}{\lambda_{0}^{2}}\right] \tau_{L}^{2}+O\left(\tau_{L}^{3}\right) .
\end{aligned}
$$

Substituting here $\lambda_{1}$ and $\lambda_{2}$, and using expansion (3.18) of $\tau_{L}$, we finally get the expression for $\mu_{a}$ that appears in Theorem 1.1. Using in (3.25) the expansions (3.9) for $\tau_{C}$ and (3.18) for $\tau_{L}$, we compute $\mu_{r}$.

Since the last assertion of Theorem 1.1 is a direct consequence of previous statements, its proof is now completed.

Acknowledgments. The authors sincerely appreciate the careful reading of the anonymous referees and their interesting suggestions that have notably improved the paper. They also want to acknowledge Jorge Galán for his invaluable suggestions and comments on a preliminary version of the manuscript, Manuel Román for his help with SPICE simulation of the circuit, and Fernando Fernández for helping with figures.

\section{REFERENCES}

[AVK66] A. A. Andronov, A. A. Vitt, And S. E. Khaikin, Theory of Oscillators, Dover, New York, 1966.

[CFPt02] V. Carmona, E. Freire, E. Ponce, and F. Torres, On simplifying and classifying piecewise-linear systems, IEEE Trans. Circuits Systems I Fund. Theory Appl., 49 (2002), pp. 609-620.

[CH82] S. N. Chow And J. K. Hale, Methods of Bifurcation Theory, Springer-Verlag, New York, Berlin, 1982.

[CWhZ93] L. ChuA, C. Wu, A. Huang, And G. Zhong, A universal circuit for studying and generating chaos-Part I: Routes to chaos, IEEE Trans. Circuits Systems I Fund. Theory Appl., 40 (1993), pp. 732-744.

[FGA84] E. Freire, L. García-Franquelo, and J. Aracil, Periodicity and chaos in an autonomous electronic oscillator, IEEE Trans. Circuits Systems, 31 (1984), pp. 237-247.

[FrgP93] E. Freire, A. J. Rodríguez-Luis, E. Gamero, and E. Ponce, A case of study for homoclinic chaos in an autonomous electronic circuit: A trip from TakensBogdanov to Hopf-ŠSil'nikov, Phys. D, 62 (1993), pp. 230-253.

[FPR99] E. Freire, E. Ponce, AND J. Ros, Limit cycle bifurcation from center in symmetric piecewise-linear systems, Internat. J. Bifur. Chaos, 9 (1999), pp. 895907.

[GK92] M. G. M. Gomes And G. P. KInG, Bistable chaos. II. Bifurcation analysis, Phys. Rev. A, 46 (1992), pp. 3100-3110.

[HBCJM91] J. J. Healey, D. S. Broomhead, K. A. Cliffe, R. Jones, and T. Mullin, The origins of chaos in a modified Van der Pol oscillator, Phys. D, 48 (1991), pp. $322-339$.

[Ke93] M. P. Kennedy, Three steps to chaos-Part II: A Chua's circuit primer, IEEE Trans. Circuits Systems I Fund. Theory Appl., 40 (1993), pp. 657-674. 
[Kr87] G. A. Kriegsmann, The rapid bifurcation of the Wien bridge oscillator, IEEE Trans. Circuits Systems, 34 (1987), pp. 1093-1096.

[Ma93] R. MAdAN, Chua's Circuit: Paradigm for Chaos, World Scientific, Singapore, 1993.

[MGhlvm03] M. B. Monagan, K. O. Geddes, K. M. Heal, G. Labahn, S. M. Vorkoetter, J. McCarron, And P. DeMarco, Maple 9 Introductory Programming Guide, Maplesoft, Waterloo, ON, 2003.

[QNPS93] T. Quarles, A. R. Newton, D. O. Pederson, And A. SangiovanniVincentelli, Spice3 Version 3f3 User's Manual, Department of Electrical Engineering and Computer Sciences, University of California Berkeley, Berkeley, CA, 1993.

[Ro03] J. Ros, Estudio del Comportamiento Dinámico de Sistemas Autónomos Tridimensionales Lineales a Trozos, Ph.D. dissertation, Universidad de Sevilla, Seville, Spain, 2003 (in Spanish).

[SYM81] R. ShinRIKI, M. Yamamoto, And S. Mori, Multimode oscillations in a modified Van der Pol oscillator containing a positive nonlinear conductance, IEEE Proc., 69 (1981), pp. 394-395.

[Wi65] J. H. Wilkinson, The Algebraic Eigenvalue Problem, Oxford University Press, Oxford, UK, 1965. 